\title{
Der Kampf um die freie Software
}

\section{Bernard Lang}

\section{(2) OpenEdition \\ 1 Journals}

Electronic version

URL: http://journals.openedition.org/sjep/565

DOI: $10.4000 /$ sjep.565

ISSN: 1663-9677

Publisher

Institut de hautes études internationales et du développement

\section{Printed version}

Date of publication: 1 novembre 2003

Number of pages: 201-205

ISSN: $1660-5926$

\section{Electronic reference}

Bernard Lang, « Der Kampf um die freie Software », Schweizerisches Jahrbuch für Entwicklungspolitik [Online], 22-2 | 2003, Online erschienen am: 10 Juni 2010, abgerufen am 08 September 2020. URL: http://journals.openedition.org/sjep/565; DOI : https://doi.org/10.4000/sjep.565 


\title{
Der Kampf um die freie Software
}

\author{
Bernard Lang*
}

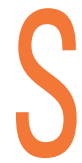

oftware-Anwendungen enthalten wesentliche Elemente der Technologie in entmaterialisierter Form. Die Kontrolle über die Software wird damit zu einem Schlüsselfaktor für die Ökonomie und die technische Entwicklung der Informationsgesellschaft. Aus der Sicht der Entwicklungsländer stellt dieser Entmaterialisierungsprozess eine ausserordentliche Chance dar, denn die Ökonomie des Immateriellen unterscheidet sich erheblich von der Ökonomie des Materiellen und ist für die Länder des Südens leichter zugänglich. Dennoch sind für die Anpassung an die neuen Gegebenheiten geeignete wirtschaftliche und technische Strukturen erforderlich. Ein Beispiel dafür ist die freie Software.

In zahlreichen Wirtschaftsbranchen wird die Entwicklung der Informationstechnologien von einer Trennung der Produkte und Tätigkeiten in einen materiellen (anpassungsfähig/programmierbar) und einen immateriellen Teil (Durchführung der Programmierung und Anpassung an spezifische Bedürfnisse) begleitet. Dies ermöglicht eine Vereinfachung und Standardisierung der materiellen Komponenten ungeachtet der letztendlichen Verwendungszwecke, was den Wettbewerb intensiviert und die Massenproduktion begünstigt. Diese beiden Faktoren bewirken eine signifikante Preissenkung für Elemente, die sich in den meisten aufstrebenden Volkswirtschaften nur schwer produzieren lassen, da sie Investitionen sowie Versorgungs-, Produktions- und Verteilungsinfrastrukturen erfordern, die in der Regel nicht verfügbar sind.

Diese häufig unvermeidliche Senkung der Importpreise ist umso mehr zu begrüssen, als sie von einer längeren Lebensdauer der Komponenten begleitet wird, da ihre Funktionen, ihr Nutzen, ihr innovativer Charakter und ihre Entwicklungsfähigkeit immer stärker auf den Informatikanwendungen, die zu ihrer Steuerung eingesetzt werden, beruhen. Nicht selten kann durch eine Neuprogrammierung der Anwendungen die Nutzung solcher Komponenten neuen Gegebenheiten angepasst und ihre Nutzungsdauer erhöht werden.

\section{Eine Schlüsselressource für arme Länder}

Damit kommt der Software entscheidende Bedeutung zu. Während die Ökonomie des Materiellen eng in Kosten- und Organisationsstrukturen eingebunden ist, gelten für die Software-Wirtschaft weitaus flexiblere Gesetze, wodurch sie leichter für alle zugänglich wird. Wie alle mittlerweile informatisierten Herstellungsprozesse für immaterielle Güter lassen sich Software-Technologien ohne

* Forschungsdirektor beim INRIA (Institut national de recherche en informatique et en automatique), $<$ www.inria.fr>, E-Mail <Bernard.Lang@inria.fr>; Vizepräsident der AFUL (Association francophone des utilisateurs de Linux et des logiciels libres), <www.aful.org $>$. 
industrielle Infrastruktur kollaborativ über das Internet entwickeln und anschliessend ohne Grenzkosten über das Web reproduzieren und verbreiten. Kostenlose Verbreitung und Nutzung können wirtschaftlich sinnvoll sein, wenn dies die Absicht der Autoren ist.

Dabei prallen zwei wirtschaftliche Tendenzen aufeinander:

- die so genannte ,proprietäre Software“, die ausschliesslich in Form von ausführbaren Anwendungen mit äusserst restriktiven Lizenzen vertrieben wird, welche ihre Nutzung stark einschränken und ein (rechtliches und technisches) Verbot beinhalten, Analysen, Anpassungen oder Verbesserungen jeder Art vorzunehmen;

- die so genannte ,freie Software“, deren Nutzung nicht eingeschränkt wird, die zusammen mit dem Quellcode (Voraussetzung für das Verständnis ihrer technischen Merkmale, für die Weiterentwicklung und für den Unterhalt) geliefert wird und welche die rechtliche und technische Möglichkeit bietet, sie zu analysieren, zu verändern, anzupassen und weiter zu vertreiben ${ }^{1}$. Denn die Entwickler von freier Software nutzen ihre Rechte, indem sie die Anwendungen der Öffentlichkeit zur Verfügung stellen².

Dies führt dazu, dass freie Software in der Regel praktisch kostenlos erhältlich ist, denn jeder Inhaber einer Kopie hat das Recht, die Anwendung weiter zu vertreiben. Proprietäre Software (die üblicherweise im Handel erhältlich ist) muss in der Regel importiert und in Devisen bezahlt werden. Damit stellt die freie Software, welche ohne Lizenz verwendet werden kann, für alle Länder, namentlich aber für die ärmeren, ein äusserst interessantes Produkt dar. Allerdings müssen sie den technischen Erfordernissen entsprechen.

\section{Drei getrennte Softwaremärkte}

Vereinfachend ausgedrückt lassen sich drei Softwaremärkte identifizieren: Personalcomputer (für den privaten oder beruflichen Gebrauch), Server sowie eingebettete Software, die das Funktionieren verschiedenster Produkte (Telefone, Autos, elektrische Geräte) gewährleistet.

In Bezug auf die Server hat sich der Einsatz freier Software bereits durchgesetzt: Das freie System Linux ist heute als Betriebssystem auf rund $30 \%$ der Unternehmensserver installiert, und $62 \%$ der Websites weltweit funktionieren mit der ebenfalls freien Software Apache. Hier ist die freie Software eindeutig die Lösung der Zukunft.

Im Bereich der Personalcomputer wird freie Software weniger häufig eingesetzt, und zwar hauptsächlich wegen der schwer veränderbaren Benutzerge-

1 Die Bereitstellung des Quellcodes zu ausschliesslichen Untersuchungszwecken ohne die Erlaubnis der freien Nutzung hat eine gegenteilige Wirkung, als dies bei der freien Software der Fall ist. Insbesondere kann in diesem Fall die unabhängige Entwicklung ähnlicher Anwendungen Anlass zu Gerichtsverfahren wegen Plagiats geben.

2 Free Software Foundation, GNU's Not Unix!, <www.fsf.org>; Open Source Initiative (OSI), The Open Source Definition, <www.opensource.org/docs/definition.php>. 
wohnheiten. Allerdings ist auch hier eine Verlagerung hin zu freier Software erkennbar, insbesondere bei den Büroanwendungen von OpenOffice.org, die für alle Systeme erhältlich sind.

Den grössten Markt stellt hingegen die in der Öffentlichkeit weniger bekannte eingebettete Software dar. Hier spielt die freie Software eine wichtige Rolle. Dies ist in einigen Fällen auf die Initiative bedeutender Konzerne zurückzuführen, denn durch die Festlegung von Standards fördern sie die Interoperabilität und somit die Zusammenarbeit und den Wettbewerb. Dabei bietet die freie Software den Vorteil, dass die Unternehmen ihre Softwareressourcen selbst kontrollieren können und nicht von der Willkür eines Lieferanten abhängig sind. Zudem müssen sie ihre Software nicht selbst entwickeln (obwohl sie natürlich einen Beitrag zur Entwicklung leisten können) und keine Lizenzgebühren bezahlen, wodurch sich ihre Wettbewerbsfähigkeit verbessert.

Die Nutzung und Veränderung von freier Software ist nicht einfach eine ideologische Entscheidung, sondern ein Hilfsmittel, welches es jedem Einzelnen ermöglicht, nach eigenem Ermessen zur Entwicklung eines gemeinsamen „Software-Erbes“ beizutragen. Dabei entsteht ein Entwicklungsmodus, der jenem der freien Wissenschaft ähnelt, welcher sich seit Jahrhunderten bewährt. Wie bei der wissenschaftlichen Forschung vermischen sich bei der freien Entwicklung die Zusammenarbeit beteiligter Akteure, der Wettbewerb unterschiedlicher Ansätze und die Kontrolle und Anerkennung durch „Peers“ (Entwickler und Nutzer), welche auf dem Wissensmarkt eine ähnlich fördernde und selektive Rolle spielen wie der Wettbewerb und die Selektion auf den traditionellen Märkten ${ }^{3}$.

\section{Vorteile für den Süden}

Dieser Produktionsmodus wirkt sich günstig auf die Qualität aus. Dies beweisen seit acht Jahren empirische Untersuchungen ${ }^{4}$, deren Resultate als Grundlage für zahlreiche Analysen dienten, welche nach einer Erklärung für diesen Erfolg suchten ${ }^{5}$. Die Transparenz, welche durch den freien Zugang einer breiten Öffentlichkeit zum Quellcode entsteht, erlaubt es, Fehler besser zu korrigieren ${ }^{6}$ und vor allen Dingen Tücken und Spionagetools zu eliminieren, die nur zu häufig in proprietären Software-Anwendungen entdeckt werden - und die in wichtigen Komponenten der Informatik-Infrastruktur besonders schwer wiegen. Zudem erfordert die dezentralisierte und vernetzte Entwicklung eine rigorose Strukturierung der Anwendungen und eine strenge Einhaltung von Standards, denn diese beiden Faktoren sind massgebend für Qualität, Unterhalt, Anpassungsfähigkeit und vor allem für die Interoperabilität und den freien Wettbewerb.

3 First Monday, Peer-reviewed Journal on the Internet, <www.firstmonday.org > (Suchbegriffe: Linux, OSS, open-source, free software, GNU, FSF).

4 Stephen Shankland (CNET News), Study Lauds Open-source Code Quality, 19 February 2003, $<$ http://news.com.com/2102-1001-985221.html>.

5 Eric S. Raymond, The Cathedral and the Bazaar, 1997, <http://catb.org/ esr/writings/cathedralbazaar>.

6 Ibid. 
Die Effizienz dieses Modells beruht aber auch auf der Tatsache, dass die Entwickler gleichzeitig Anwender sind, und dass sich unter diesen Akteuren neben Grosskonzernen (SUN, IBM, HP, EDF), KMU (O'Reilly, Trolltech), Verbänden (Debian, KDE), Gebietskörperschaften (Estremadura) und Behörden (Deutschland, Frankreich) auch Einzelpersonen (R. Stallman, L. Torvalds) befinden. Die wirtschaftlichen Beweggründe sind vielfältig. $\mathrm{Zu}$ den wichtigsten gehören die technische Qualität, die Kosteneinsparungen, welche durch ein gemeinschaftliches Vorgehen ohne äussere Kontrolle ermöglicht werden, die grössere Unabhängigkeit bei der Nutzung einer strategisch wichtigen Ressource sowie die Förderung ergänzender wirtschaftlicher Tätigkeiten. Angesichts dessen haben die politischen Behörden weltweit ein grosses Interesse daran, dass die freie Software gefördert wird ${ }^{7}$.

Den Entwicklungsländern bietet die freie Software zahlreiche Vorteile. Aus wirtschaftlicher Sicht ermöglicht sie bedeutende Einsparungen von Devisen, die für Lizenzen und Dienstleistungen ausgegeben werden müssen. Denn Leistungen, die früher importiert werden mussten, werden nun durch die Binnenwirtschaft erbracht. Die Entstehung einer lokalen Industrie, welche die Software an die Bedürfnisse des Landes anpasst, begünstigt die technologische Unabhängigkeit und die wirtschaftliche Entwicklung. Global betrachtet stellt die Verfügbarkeit der Quellcodes eine Bildungsressource dar, die es ermöglicht, technisches Know-how zu erwerben, das jenem der übrigen Ländern ebenbürtig ist. Nicht zuletzt bietet die Gleichstellung in Bezug auf Zugang und Bildung inländischen Technikern und Entwicklern die Möglichkeit, sich in das weltweite technologische Beziehungsnetz zu integrieren und selbst einen Beitrag zu leisten.

Die Rolle der Software als Träger von Kommunikation, Wachstum und Kultur ist bereits heute von zentraler Bedeutung, und sie wird künftig weiter an Wichtigkeit gewinnen. Das ist insofern als Chance zu betrachten, als alle Länder gegenüber den immateriellen Ressourcen gleichgestellt sind - unter der Voraussetzung allerdings, dass sie diese frei und unabhängig nutzen können und die inhärente Flexibilität immaterieller Errungenschaften verwenden können, um sie ihren eigenen Bedürfnissen und Anforderungen anzupassen. Die freie Software bietet diese Möglichkeit im Hinblick auf Medien und Datenträger. Allgemein wird dies auch durch die „freien Inhalte“ gewährleistet, das heisst die intellektuellen Ressourcen - künstlerischer, erzieherischer, technischer oder wissenschaftlicher Natur -, die von ihren Urhebern der Allgemeinheit frei verfügbar gemacht werden. Zusammen leisten freie Software und freie Inhalte, in einem natürlichen Rahmen der Zusammenarbeit unter Gleichgestellten, einen Beitrag zur Unabhängigkeit und zur kulturellen Vielfalt: Integration ohne Entfremdung.

7 GrULiC (Grupo de Usuarios de Linux de Córdoba), Proposal for the Use of Open Technologies in the Government/Proposición para el uso de Tecnologías Abiertas en el Estado, 03/02/03, <http://proposicion.org.ar>; Sam Kritikos, The State of Open Source (SOS), 3 February 2003, <www.gnacademy.org/twiki/bin/view/SOS>; Antwort des peruanischen Abgeordneten Edgar Villanueva Nuñez an Microsoft (in 10 Sprachen), 8. April 2002, sowie weitere Dokumente <www.pimiento.linux.com/ peru $2 \mathrm{~ms}>$. 
Die Entwicklung freier Software beruht namentlich auf dem Urheberrecht, welches dank Lizenzen wie beispielsweise die General Public Licence - $\mathrm{GPL}^{8}$ eine unbemerkte Aneignung ausschliesst. Das Urheberrecht dient traditionell dem Schutz immaterieller Errungenschaften, zu denen auch die Software gehört. Das Urheberrecht ist unentgeltlich und somit auch für Erzeugnisse geeignet, die ohne Erwerbszweck entwickelt und verbreitet werden.

Allerdings wird dieser Sachverhalt von verschiedenen Interessensgruppen, welche eine Patentierbarkeit von Softwaretechniken fordern, in Frage gestellt. Für gewisse bedeutende Herausgeber von proprietärer Software stellt das Patent eine wirksame Waffe gegen neue Konkurrenten (Unternehmen oder Länder) dar. In den so genannten „Halloween Documents“9 erläuterte das Unternehmen Microsoft seine Vision der freien Software und lieferte aus seiner Sicht eine Analyse ihrer Wirkung. Laut Microsoft stellt die Patentierbarkeit von Software ein wirksames Mittel dar, um dem Vormarsch der freien Software Einhalt zu gebieten. In der Tat können durch eine nicht gewinnorientierte Entwicklung und Verbreitung weder die Kosten für die Patentierung noch jene im Zusammenhang mit allfälligen Rechtsstreiten gedeckt werden. Streitigkeiten jedoch lassen sich kaum vermeiden: Während im Urheberrecht Nachahmungen die Ausnahme - und nur selten unbeabsichtigt - sind, ist es durchaus üblich, dass patentierte Erfindungen unbeabsichtigt und unbewusst nachgeahmt werden - mit teilweise dramatischen finanziellen Folgen.

Die Entwicklung freier Software ist aus technischer und wirtschaftlicher Sicht effizient und stellt für die Länder des Südens - und in geringerem Masse auch für den Norden - eine bedeutende Chance dar. Aber wie in zahlreichen anderen Bereichen besteht auch in Bezug auf die freie Software die Gefahr, dass die Entwicklungsländer als Erste der exzessiven, unkontrollierten und ungerechtfertigten Ausdehnung der geistigen Eigentumsrechte zum Opfer fallen. Im Rahmen der internationalen Verhandlungen, insbesondere in der Welthandelsorganisation (WTO) und der Weltorganisation für geistiges Eigentum (WIPO), ist eine weit reichende Mobilisierung in diesen Fragen unverzichtbar, denn sie sind ausschlaggebend für die Beteiligung aller an der Informationsgesellschaft und bestimmen den wirtschaftlichen, gesellschaftlichen und kulturellen Erfolg der Informationsgesellschaft als Ganzes. 